\title{
Turning to Thyroid Disease in Pregnant Women
}

\author{
Stine Linding Andersen ${ }^{a} \quad$ Stig Andersen ${ }^{b, c}$ \\ ${ }^{a}$ Department of Clinical Biochemistry, Aalborg University Hospital, Aalborg, Denmark; ${ }^{b}$ Department of Geriatrics, \\ Aalborg University Hospital, Aalborg, Denmark; ' ${ }^{C}$ epartment of Clinical Medicine, Aalborg University, Aalborg, \\ Denmark
}

\section{Key Points}

- Overt thyroid disease should be diagnosed and treated in nonpregnant and pregnant patients.

- Subclinical thyroid disease management is debatable in nonpregnant and pregnant patients.

- Smaller aberrations in thyroid function have drawn much attention in pregnant women, specifically.

- A main focus on maternal T4 in pregnancy emerged before TSH assays were available.

- With the advancement of assays, the concept of "isolated hypothyroxinemia" emerged.

- No effect of treating such smaller aberrations in maternal thyroid function appear from large RCTs.

- A turn towards thyroid disease in pregnant women may be justified.

\section{Keywords}

Thyroid function · Pregnancy · Hyperthyroidism •

Hypothyroidism

\section{Abstract}

Thyroid disease in pregnant women needs attention from a clinical and scientific standpoint due to the potential severe adverse consequences. It is well-established that overt thyroid disease in pregnant women should be treated to prevent maternal and fetal complications, but routine testing for overt thyroid function test abnormalities has not been implemented. In contrast, the scientific focus has shifted towards smaller aberrations in maternal thyroid function including subclinical thyroid disease and isolated deviations in maternal thyroxine. In this focused review, we touch upon the assessment of maternal thyroid function in pregnancy and how the historical advancements in thyroid function tests parallel with the thyroid function test abnormalities described. Furthermore, we discuss how the scientific focus has evolved and how the field could turn in view of the existing discrepancies between results of observational studies and randomized controlled trials.

(C) 2020 European Thyroid Association Published by S. Karger AG, Basel

\section{Introduction}

Maternal thyroid function in pregnancy has long been a focus of scientific work and a critical concern in clinical care. The description of cretinism in areas with severe iodine deficiency provided the first historical evidence of the consequences of abnormalities in maternal thyroid function in pregnancy $[1,2]$. The important role of maternal thyroid hormones in fetal brain development was further substantiated from the paucity of neurological 
Fig. 1. Historical overview of the introduction of methods used for the assessment of human thyroid function in clinical practice.

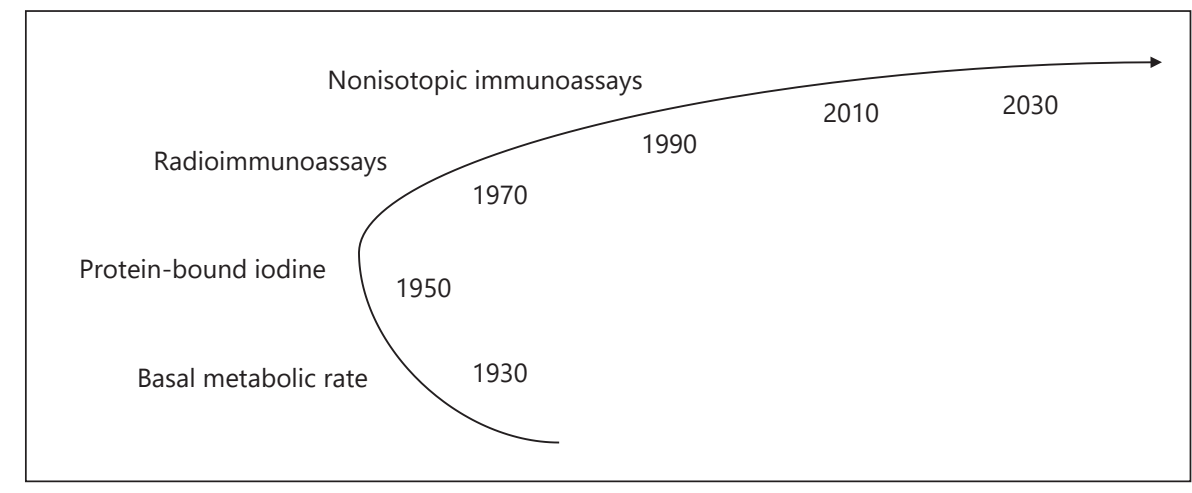

symptoms in children with congenital hypothyroidism who received prompt treatment immediately after birth [3] and from the measurement of thyroxine (T4) in cord blood from newborns who were unable to synthesize thyroid hormones due to a defect in organification [4]. Consensus is clear on the adverse consequences of untreated congenital hypothyroidism and the benefits of treatment, and neonatal screening has been implemented worldwide for decades [3]. On the other hand, clinical guidelines conclude that evidence for or against universal screening for maternal thyroid disease in pregnancy is insufficient at present $[5,6]$. The lack of evidence is not caused by a limited number of studies since the number of publications within clinical thyroidology is high and is rapidly evolving [7]. It is also well-established in clinical guidelines that overt thyroid disease in pregnant women should be treated to prevent maternal and fetal complications [6]. However, a gap in the knowledge persists as to the uncertainties regarding the consequences of smaller aberrations in maternal thyroid function $[5,6]$.

In this focused review, we discuss the continuum of maternal thyroid function test abnormalities in pregnancy. Specifically, we describe the assessment of maternal thyroid function in pregnancy, the management of overt and subclinical thyroid disease, and the historical turning towards a focus on smaller aberrations. Moreover, we touch upon the disparity in results of observational studies and randomized controlled trials (RCTs) and we speculate on future perspectives within the field.

\section{Assessment of Maternal Thyroid Function}

As in nonpregnant individuals, abnormal thyroid function in pregnant women includes a continuum from excess to insufficient production of thyroid hormone.
Available methods for the assessment of thyroid function have advanced considerably during the last century (Fig. 1) [8]. It was not until the 1970s that the radioimmunoassay for the measurement of TSH was introduced and prior to that point in time, biochemical assessment of thyroid function relied on the measurement of proteinbound iodine (PBI). PBI was considered an indirect measure of circulating thyroid hormones and the subsequent butanol-extracted iodine (BEI) was developed to consider mainly iodine bound to T4 [9].

It is well-established that the primary test for the screening of thyroid dysfunction in nonpregnant individuals is TSH. The superiority of TSH relates to the loglinear relationship between TSH and free T4, meaning that small deviations in free T4 will provoke a much larger response in TSH [8]. The most commonly used method for the measurement of TSH in clinical laboratories today is an automatic nonisotopic immunoassay (Fig. 1). The sensitivity of the assay has improved, and the current third-generation assay has a functional sensitivity of 0.01 $\mathrm{mIU} / \mathrm{L}[8,9]$. In addition to the analytical performance, the biological variation of TSH is of importance. Studies on nonpregnant individuals [10] and pregnant women [11] have shown that the intraindividual variation of TSH is narrow as opposed to the interindividual variation, and the low index of individuality challenges the use of population-based reference ranges. TSH is also the first-line thyroid function test for the diagnosis of thyroid dysfunction in pregnant women, but the physiological alterations in pregnancy necessitate the use of a pregnancy-specific reference range $[12,13]$. It is important to notice that the use of TSH for the diagnosis of thyroid dysfunction relies on a balance within the hypothalamic-pituitary-thyroid axis. This equilibrium may be altered in individuals suffering from nonthyroidal illness. Furthermore, TSH cannot stand alone for the assessment of thyroid function in 
Fig. 2. The association between maternal TSH in early pregnancy and child verbal IQ in a study on 1,153 Danish pregnant women and their 5-year-old children. Point estimates are adjusted mean difference with 95\% confidence interval. The adjusted model included maternal IQ, pre-pregnancy body mass index, the child's age at testing, and the testing psychologist. Reproduced with the permission of Andersen et al. [24].

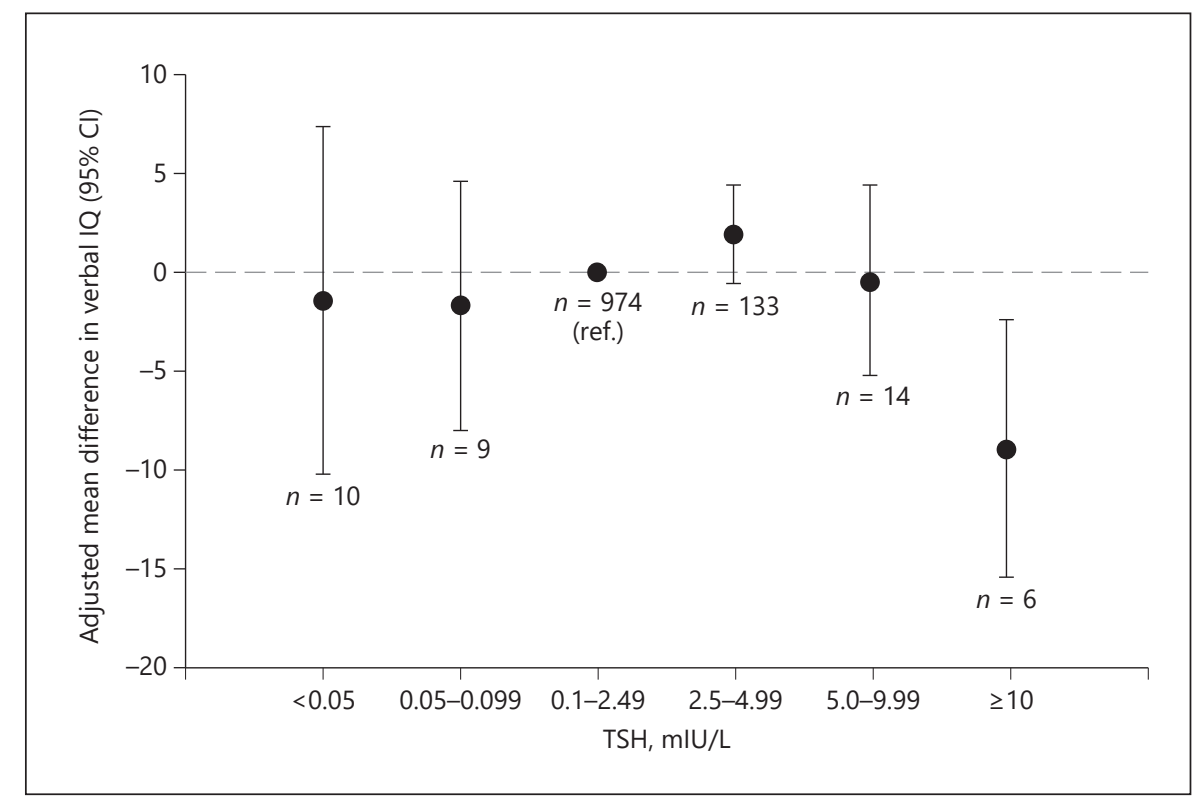

pregnant women who receive current treatment with levothyroxine or antithyroid drugs. In such patients, a measure of the peripheral thyroid hormone concentration should also be considered. The need for accurate assessment is illustrated by the potential severe consequences for the fetus [14].

The estrogen-mediated increase in thyroxin-binding globulin (TBG) in pregnancy is followed by an increase in total thyroid hormone concentrations, and pregnancy-specific reference ranges for total T4 and total triiodothyronine (T3) should be used [6]. Much emphasis has been on the use of free thyroid hormone concentrations to overcome the physiological alterations in protein binding. However, the method used for the measurement of free T4 and T3 in clinical laboratories is the nonisotopic immunoassay, which involves an indirect approach with no initial separation of the free and protein-bound hormone [15]. The measurements are thus prone to alterations in protein binding, also in pregnant women, and pregnancy- and method-specific reference ranges should be used [16-18]. The routine availability of total and/or free thyroid hormone measurements varies between clinical laboratories. It has been proposed that total T4 is inferior to free T4 for the assessment of maternal thyroid function in pregnancy [19]. However, an emphasis on TSH for the diagnosis of thyroid disease in nonpregnant as well as pregnant individuals, and on the importance of method- and pregnancy week-specific reference ranges for all thyroid function tests used in pregnant women may be more relevant than focusing on either the total or the free thyroid hormone concentration.

\section{Overt Thyroid Disease}

Overt thyroid dysfunction in pregnant women is defined by TSH and T4 outside of the pregnancy- and method-specific reference ranges [6]. Clinical guidelines unanimously state that overt hyperthyroidism caused by Graves' disease as well as overt hypothyroidism in pregnant women should be treated, and that conducting RCTs to evaluate the effect of treating such abnormalities would be unethical [6]. Overt thyroid disease in pregnant women has been associated with pregnancy complications as well as adverse neuropsychological outcomes in the child, and a hypothesis of fetal programming by maternal thyroid disease has been proposed [20,21]. Fetal programming is a long-standing concept that links in utero exposure with the development of disease in later life. The theory is not exclusively related to the field of thyroid disease [21]. Numerous mechanisms have been proposed, and, for the hypothesis of fetal programming by maternal thyroid disease, a focus on fetal brain development is biologically plausible. Prospective studies with randomization to treatment of overt thyroid disease in pregnant women should not be performed. However, evidence from retrospective observational studies has demonstrated that the 
risk of adverse outcomes is particularly high when maternal overt thyroid disease is left undiagnosed and untreated [22]. In the hallmark study by Haddow et al. [23] published in 1999, untreated maternal hypothyroidism $(n=$ 48) evaluated from the measurement of TSH in pregnancy week 17 (TSH range $\sim 5-90 \mathrm{mIU} / \mathrm{L}$ ) was associated with a lower intelligence quotient (IQ) in the child at the age of 7-9 years (a mean full-scale IQ score of 100) compared to nonexposed controls ( $n=124$; a mean full-scale IQ score of 107), whereas no difference in child IQ was observed when the maternal hypothyroidism had been treated $(n=$ 14; a mean full-scale IQ score of 111) during the pregnancy. In line with these results, in the Danish National Birth Cohort, maternal TSH $\geq 10 \mathrm{mIU} / \mathrm{L}$ assessed in median pregnancy week 9 was a risk factor for low verbal IQ in the child at 5 years of age (Fig. 2) [24]. Although this study included only a small number of exposed children, the results substantiate the association with marked maternal undiagnosed and untreated hypothyroidism [24].

IQ is a standardized method for evaluating intelligence. It is a global test that does not tell you directly about specific underlying brain morphological abnormalities. However, studies on patients with focal brain damage have provided clues to the relationship between different measures of intelligence and neuroanatomic alterations [25, 26]. Adding to this, an investigation in the Generation $R$ study included magnetic resonance imaging (MRI) of the brain in 1,981 children at a median age of 10 years; a $\mathrm{U}$ shaped relationship between maternal TSH and child total and cortical grey matter volume was revealed, and this was most evident when maternal thyroid function was assessed early in the pregnancy [27]. In most studies, maternal thyroid function has been assessed from a single measurement and may not reflect the thyroid function throughout the pregnancy. Notably, the biological variation of thyroid function tests is of the same magnitude in nonpregnant [10] and pregnant [11] individuals. The more pronounced the TSH abnormality, the more likely the thyroid function abnormality will persist if not treated in nonpregnant individuals [28]. In pregnant women, it was observed in studies from Israel [29] and China [30] that $<25 \%$ of maternal thyroid function abnormalities persisted until the third trimester, but persistency was dependent on maternal TSH, T4, and thyroid peroxidase antibodies (TPO-Ab). On the other hand, 2 Danish studies $[31,32]$ examined the persistency after the pregnancy, and showed that $>50 \%$ of women clinically diagnosed and treated for hypothyroidism in the years following the pregnancy had had undetected overt or subclinical hypothyroidism in the early pregnancy [31].

\section{Subclinical Thyroid Disease}

The clinical focus on overt thyroid disease and the recommendation of treatment for pregnant women is compatible with the clinical approach for nonpregnant individuals. Regarding subclinical thyroid function test abnormalities with abnormal TSH in combination with a T4 within the reference range, the clinical management is less clear and debatable in both pregnant and nonpregnant individuals $[5,6,28,33]$. Subclinical hyperthyroidism has not been associated with pregnancy complications [34] and treatment is not recommended in pregnancy [6]. On the other hand, recommendations for treatment of maternal subclinical hypothyroidism include the assessment of the level of TSH and the presence of TPO-Ab $[5,6]$. Levothyroxine is recommended in TPO-Ab-negative pregnant women when maternal TSH is $>10 \mathrm{mIU} / \mathrm{L}$, and it may also be considered if TSH is above the pregnancy week-specific upper reference limit. On the other hand, in TPO-Ab-positive women, levothyroxine treatment is recommended when maternal TSH is above the upper pregnancy week-specific reference range and may be considered if TSH is $>2.5$ mIU/L [6]. A substantial number of observational studies have addressed the impact of subclinical hypothyroidism in pregnant women and they point towards adverse complications (e.g., miscarriage, preterm birth, pre-eclampsia, and gestational diabetes), especially in TPO-Ab-positive women and in women with a marked elevation of TSH $[6,35]$. On the other hand, a limited number of observational studies evaluated the impact on child neurocognitive development and showed inconsistent results $[5,6]$. Despite the diversity in observational findings, the field has moved forward to the performance of RCTs that evaluated the effect of levothyroxine treatment in women with subclinical hypothyroidism and/or TPO-Ab on the outcomes of pregnancy complications and child neurocognitive development [36-43] (Table 1). Notably, the smallest studies reported a beneficial effect of treatment on pregnancy complications, whereas the large studies evaluating child neurocognitive development showed no effect (Table 1). It should be kept in mind that a number of methodological aspects may be of importance in the evaluation of RCTs. The timing of the onset of treatment, the dose of levothyroxine, and the age of the child at the outcome assessment have been discussed [44, 45]. However, a recent follow-up of the Controlled Antenatal Thyroid Screening (CATS) study addressed some of these concerns and supported the conclusions $[46,47]$. 
Table 1. RCTs on the effect of treating smaller aberrations in maternal thyroid function and/or thyroid autoimmunity

\begin{tabular}{|c|c|c|c|c|c|c|c|}
\hline First author [ref.] & Year & Country & Exposure & $N^{1}$ & Outcome & L-T4 dose/day & L-T4 start \\
\hline \multicolumn{8}{|l|}{ Effect of treatment } \\
\hline Negro [36] & 2006 & Italy & TPO-Ab-positive, euthyroid & 115 & Miscarriage and preterm birth & $0.5-1.0 \mu \mathrm{g} / \mathrm{kg}$ & Week 10.4 \\
\hline Negro [37] & 2010 & Italy & TPO-Ab-positive, TSH >2.5 mIU/L & 118 & Adverse pregnancy outcomes & Unknown & Week 12 \\
\hline Nazarpour [38] & 2017 & Iran & TPO-Ab-positive, euthyroid & 131 & Miscarriage and preterm birth & $0.5-1.0 \mu \mathrm{g} / \mathrm{kg}$ & Week 11.4 \\
\hline \multicolumn{8}{|l|}{ No effect of treatment } \\
\hline Lazarus [39] & 2012 & UK/Italy & Subclinical hypo/hypothyroxinemia & $496 / 499$ & Child IQ at 3 years of age & $150 \mu \mathrm{g}$ & Week 13.3 \\
\hline Casey [40] & 2017 & USA & Subclinical hypo/hypothyroxinemia & $677 / 526$ & Child IQ at 5 years of age & 50 or $100 \mu \mathrm{g}$ & Week 16.7 \\
\hline Wang $[41]^{2}$ & 2017 & China & TPO-Ab-positive, euthyroid & 600 & Miscarriage and live-birth rate & 25 or $50 \mu \mathrm{g}$ & Pre-pregnancy \\
\hline Nazarpour [42] & 2018 & Iran & TPO-Ab-negative, TSH >2.5 mIU/L & 366 & Miscarriage and preterm birth & $1.0 \mu \mathrm{g} / \mathrm{kg}$ & Week 11.4 \\
\hline Dhillon-Smith $[43]^{3}$ & 2019 & USA & TPO-Ab-positive, euthyroid & 952 & Live-birth rate & $50 \mu \mathrm{g}$ & Pre-pregnancy \\
\hline
\end{tabular}

RCTs, randomized controlled trials; hypo, hypothyroidism; IQ, intelligence quotient; L-T4, levothyroxine; TPO-Ab, thyroid peroxidase antibodies.

${ }^{1}$ Total number of women randomized; ${ }^{2}$ women undergoing in vitro fertilization; ${ }^{3}$ women with a history of miscarriage or infertility.

Table 2. Historical overview of review papers on the consequences of maternal hypothyroxinemia in pregnancy

\begin{tabular}{lll}
\hline Year & First author [ref.] & Title $^{1}$ \\
\hline 1972 & Man [49] & Thyroid function in pregnancy and infancy. Maternal hypothyroxinemia and retardation of progeny \\
1992 & Sinha [50] & Maternal hypothyroxinemia and brain development: a hypothetical control system governing fetal exposure to maternal thyroid hormones \\
2000 & Glinoer [51] & The potential repercussions of maternal, fetal, and neonatal hypothyroxinemia on the progeny \\
2000 & Morreale de Escobar [52] & Is neuropsychological development related to maternal hypothyroidism or to maternal hypothyroxinemia? \\
2003 & Morreale de Escobar [53] & Maternal hypothyroxinemia versus hypothyroidism and potential neurodevelopment. Alterations of her offspring \\
2007 & Román [54] & Autism: transient in utero hypothyroxinemia related to maternal flavonoid ingestion during pregnancy and to other environmental antithyroid agents \\
2011 & Negro [55] & Hypothyroxinemia and pregnancy \\
2013 & Henrichs [56] & Maternal hypothyroxinemia and effects on cognitive functioning in childhood: how and why? \\
2015 & Furnica [57] & Update on new controversy in endocrinology: isolated maternal hypothyroxinemia \\
2015 & Chan [58] & Optimal management of hypothyroidism, hypothyroxinemia, and euthyroid TPO antibody positivity preconception and in pregnancy \\
2016 & Lazarus [59] & Hypothyroxinaemia and brain development \\
2016 & Min [60] & Maternal hypothyroxinemia-induced neurodevelopmental impairments in the progeny \\
2017 & Dosiou [61] & Isolated maternal hypothyroxinemia during pregnancy: knowns and unknowns
\end{tabular}

${ }^{1}$ The review papers were identified in the PubMed database using the term: (hypothyrox ${ }^{\star}$ Title]) AND pregnancy AND review.

Importantly, a concern was raised about adverse effects related to overtreatment of maternal thyroid function $[46,47]$. Levothyroxine is a replacement therapy and is, in general, considered safe. However, a concern about levothyroxine treatment of pregnant women was also highlighted in a large retrospective study from the USA [48]. In this study, treatment of maternal subclinical hypothyroidism in pregnancy was associated with a lower risk of pregnancy loss, especially when maternal TSH was $>4.0 \mathrm{mIU} / \mathrm{L}$, but a higher risk of other adverse complications was observed (preterm birth, pre-eclampsia, and gestational diabetes) [48]. As concluded by the authors, the findings are of importance for clinical decision-making upon the initiation of treatment and should be addressed in additional studies.

\section{The Turning towards Smaller Abnormalities}

A third category of thyroid function test abnormalities has been considered in pregnant women (Table 2), but it has not been a matter of such extensive clinical attention in nonpregnant adults. This is hypothyroxinemia, which is defined by a low maternal T4, and when the term "isolated maternal hypothyroxinemia" is used, it refers to a low T4 in combination with a normal TSH $[5,6]$. One may speculate on how this turn towards a focus on smaller abnormalities in maternal thyroid function emerged? It is not part of the general concept of clinical hypothyroidism; however, an increasing number of review papers [49-61] (Table 2), observational studies [5, 6, 35], and RCTs $[39,40]$ (Table 1) have focused on this entity in relation to pregnant women. 
The PubMed database covers publication from the 1950s to the present, and the first review paper on maternal hypothyroxinemia in pregnancy identified in this database was published in 1972 by Evelyn Man [49] (Table 2 ). In this paper, the author reviewed the results of her own investigations performed in the period 1962-1967, at a time when maternal thyroid function was assessed from the measurement of BEI as a proxy for T4. A total of 131 pregnant women had low BEI in pregnancy and were classified as having hypothyroxinemia, and then stratified according to whether they had been adequately treated during pregnancy. Compared with euthyroid controls, children born to mothers with inadequately treated hypothyroxinemia had a lower IQ at 4 and 7 years of age. Notably, this investigation was performed in the 1960s prior to the introduction of the TSH method (Fig. 1), and it may be speculated that some of the women not only suffered from isolated hypothyroxinemia but may actually have had overt hypothyroidism. However, the entity hypothyroxinemia was proposed from the measurement of BEI, which was used as a crude measure of thyroid function.

In the 1960s, another area that drew attention to maternal T4 was the description of endemic cretinism in areas with severe iodine deficiency $[1,2]$. A specific hypothesis for the development of neurological and myxedematous cretinism was proposed in 1993 [62]. One conclusion was that there was ample evidence to suggest that maternal and fetal hypothyroxinemia caused by iodine deficiency was a major pathogenic factor for the development of neurological cretinism [62]. The role of maternal thyroid function was evident from the prevention of cretinism by iodine supplementation given to the mother in pregnancy and from the measure of maternal thyroid function in pregnancy $[1,2]$. However, at the time of the original investigations, PBI [1, 2], and subsequently T4 [63], were the methods available, and maternal hypothyroxinemia was the logical term to consider. In 1999, Pop et al. [64] published an original investigation showing that low maternal free $\mathrm{T} 4$ assessed in pregnancy week 12 was associated with impaired psychomotor function of the infant. TSH methods were available at this time (Fig. 1) and maternal TSH was measured in this cohort using a third-generation nonisotopic immune assay, but it was not used for classification of maternal thyroid function. Thus, the studies mentioned so far considered maternal T4 irrespective of maternal TSH, and some of the women are likely to have suffered from overt hypothyroidism in pregnancy.
A comprehensive review paper by Morreale de Escobar et al. [52] published in 2000 (Table 2) questioned whether the neuropsychological development of the offspring was related to maternal hypothyroidism or hypothyroxinemia. In this review, the author summarized evidence from human and experimental studies, and suggested that a low maternal free T4 (irrespective of TSH) was a risk factor for impaired neuropsychological development of the fetus. A considerable number of studies were available at this time, but the majority were performed before TSH measurement was possible. The linkage between experimental findings and the clinical focus on free T4 in pregnant women put forward by the author remains uncertain. Still, the review drew more attention to the role of maternal T4, and since the year 2000, an increasing number of original studies and review papers (Table 2) have focused on the role of maternal hypothyroxinemia in pregnancy. With the possibility of routine measurement of TSH, the definition has emerged to isolated maternal hypothyroxinemia, i.e., low T4 in the absence of elevated TSH. However, the definition of low T4 is not consistent, and it varies between studies according to whether a 2.5-; 5-, or even a 10 -percentile reference cut-off is used [61].

\section{Association or Causation?}

It is 20 years since the review by Morreale de Escobar et al. [52] was published. An intriguing question is how the interpretation of smaller aberrations in maternal thyroid function has evolved since then. A large number of observational studies have been performed evaluating the association between maternal hypothyroxinemia and outcomes of pregnancy and child development $[5,6]$. Overall, the findings suggest an association with adverse outcomes of pregnancy including impaired neurocognitive development of the child $[5,6]$. However, this is in contrast to the lack of effect of treatment in most of the RCTs performed (Table 1). In particular, the 2 large RCTs designed to evaluate the effect of treating maternal hypothyroxinemia in pregnancy in relation to child IQ did not show any effect of treatment $[39,40]$.

As previously specified, a number of methodological aspects may be of importance when interpreting the RCT results. However, the discrepancy between observational findings and RCTs may question the causal role of smaller aberrations in maternal thyroid function in pregnancy with regard to the associations observed. Notably, in oth- 
er research fields, a disparity exists between observational findings and RCT results, e.g., the protective role of omega-3 fatty acids on the risk of cardiovascular disease described in observational studies has not been replicated in RCTs [65], nor has the protective role of vitamin D supplementation on the risk of cardiovascular disease and cancer [66]. Finally, the discrepancy with observational studies evaluating the effect of hormone replacement therapy on the risk of coronary heart disease and breast cancer is a well-known epidemiological example [67]. Observational studies are prone to confounding [68]. Considering the association between smaller abnormalities in maternal thyroid function and outcomes of pregnancy, it is interesting to observe an increasing burden of evidence suggesting that maternal free T4 associates with a number of maternal characteristics that are not necessarily directly related to the thyroid gland. Thus, the level of free T4 has been associated with maternal obesity and adverse metabolic parameters [69], gestational diabetes [70], and maternal iron status [71]. Furthermore, the lack of specificity in the observational findings, e.g., the dependency on the sex of the child and whether the evaluation was performed by the teacher or the parent, may question the underlying mechanisms of the associations observed [24]. In addition, it has been speculated whether alterations in maternal free $\mathrm{T} 4 \mathrm{reflect}$ the thyroid status of the pregnant woman, or if they are a marker of placental function or other maternal characteristics; this could confound the association observed [22].

\section{Perspectives}

The field of thyroid disease in pregnancy is at a crossroad between observational findings and the results of RCTs considering the impact of smaller aberrations in maternal thyroid function. From a historical review, it appears that the scientific focus has taken a turn towards isolated deviations in maternal T4, even though such abnormalities are not part of the clinical spectrum of thyroid disease in nonpregnant individuals. The lack of treatment effects in large RCTs is food for thought. One may speculate on the methodological aspects, such as study design, and the definition of exposure and outcome where some features are yet to be clarified. This includes the reference range for the assessment of maternal thyroid function in pregnancy, assay performance, and early inclusion for the initiation of treatment. It also includes details on the methods used for the assessment of child neurodevelopment by neuropsychological testing, brain morphology, and long-term outcomes. That being said, the discrepancy between the results of numerous observational studies and large, expensive RCTs should encourage further consideration. It is unanimously stated in clinical guidelines that overt thyroid disease in pregnant women should be treated [6]. The detection of such undiagnosed overt thyroid function test abnormalities would identify a larger number of cases than the current screening for congenital hypothyroidism [22], but no routine testing of pregnant women has been hitherto implemented.

Perhaps now is the time for the field to turn the focus towards thyroid disease in pregnant women. Unresolved matters include the benefits and risks of the routine detection of maternal overt thyroid disease. The level of abnormality of thyroid function also needs attention, to be able to move forward, e.g., by stratification of when and how to diagnose and treat maternal subclinical thyroid disease. In parallel with this focus on thyroid disease, future research should enhance our understanding of smaller abnormalities in maternal thyroid function as well as thyroid autoimmunity, per se, and the interaction with other determinants prior to the performance of further large RCTs. Observational studies have their advantages. A further enhancement of the definition of exposure and outcome, combined with the use of new and alternative epidemiological methods (e.g., Mendelian randomization, instrumental variables, twin studies, and negative control exposure), has come about for the determination of causation in the field of thyroid disease in nonpregnant individuals [72], and this may strengthen the level of evidence.

\section{Conclusion}

The consequences of lack of treatment of maternal thyroid disease in pregnancy may be severe. Scientific focus has turned towards the impact of smaller aberrations in maternal thyroid function. Treatment of such small abnormalities has shown no effect in large RCTs, in contrast to observational findings. A scientific turn towards thyroid disease and the benefits and risk of routine testing and treatment of such abnormalities may be justified.

\section{Disclosure Statement}

The authors have no conflict of interests to declare. 


\section{Funding Sources}

There was no funding.

\section{Author Contributions}

Stine Linding Andersen conceptualized and drafted the manuscript. Stig Andersen critically reviewed, discussed, and commented on the manuscript.

\section{References}

1 Choufoer JC, Vanrhijn M, Querido A. Endemic goiter in Western New Guinea. II. Clinical picture, incidence and pathogenesis of endemic cretinism. J Clin Endocrinol Metab. 1965 Mar;25(3):385-402.

2 Pharoah PO, Buttfield IH, Hetzel BS. Neurological damage to the fetus resulting from severe iodine deficiency during pregnancy. Lancet. 1971 Feb;1(7694):308-10.

3 Grüters A, Krude H. Detection and treatment of congenital hypothyroidism. Nat Rev Endocrinol. 2011 Oct;8(2):104-13.

4 Vulsma T, Gons MH, de Vijlder JJ. Maternalfetal transfer of thyroxine in congenital hypothyroidism due to a total organification defect or thyroid agenesis. N Engl J Med. 1989 Jul; 321(1):13-6.

5 Lazarus J, Brown RS, Daumerie C, Hubalewska-Dydejczyk A, Negro R, Vaidya B. 2014 European thyroid association guidelines for the management of subclinical hypothyroidism in pregnancy and in children. Eur Thyroid J. 2014 Jun;3(2):76-94.

6 Alexander EK, Pearce EN, Brent GA, Brown RS, Chen H, Dosiou C, et al. 2017 Guidelines of the American Thyroid Association for the Diagnosis and Management of Thyroid Disease During Pregnancy and the Postpartum. Thyroid. 2017 Mar;27(3):315-89.

7 Cooper DS, Anton B. The Decade in Clinical Thyroid Disease: An Analysis of Published Literature. Thyroid. 2016 Aug;26(8):9931003.

8 Spencer C [Internet]. Thyroid function tests: assay of thyroid hormones and related substances [cited 2019 Dec 12]. Available from: www.thyroidmanager.com

9 Kuyl JM. The evolution of thyroid function tests. J Endocrinol Metab Diabetes South Africa. 2015;20(2):11-6.

10 Andersen S, Bruun NH, Pedersen KM, Laurberg P. Biologic variation is important for interpretation of thyroid function tests. Thyroid. 2003 Nov; 13(11):1069-78.

11 Boas M, Forman JL, Juul A, Feldt-Rasmussen U, Skakkebaek NE, Hilsted L, et al. Narrow intra-individual variation of maternal thyroid function in pregnancy based on a longitudinal study on 132 women. Eur J Endocrinol. 2009 Dec;161(6):903-10.

12 Laurberg P, Andersen SL, Hindersson P, Nohr EA, Olsen J. Dynamics and Predictors of Serum TSH and fT4 Reference Limits in Early Pregnancy: A Study Within the Danish National Birth Cohort. J Clin Endocrinol Metab. 2016 Jun;101(6):2484-92.
13 Andersen SL, Andersen S, Carlé A, Christensen PA, Handberg A, Karmisholt J, et al. Pregnancy Week-Specific Reference Ranges for Thyrotropin and Free Thyroxine in the North Denmark Region Pregnancy Cohort. Thyroid. 2019 Mar;29(3):430-8.

14 Feldt-Rasmussen U, Bliddal S, Rasmussen AK, Boas M, Hilsted L, Main K. Challenges in interpretation of thyroid function tests in pregnant women with autoimmune thyroid disease. J Thyroid Res. 2011 Mar;2011(Mar): 598712.

15 Laurberg P, Andersen SL. ENDOCRINOLOGY IN PREGNANCY: pregnancy and the incidence, diagnosing and therapy of Graves' disease. Eur J Endocrinol. 2016 Nov; 175(5):R219-30.

16 Lee RH, Spencer CA, Mestman JH, Miller EA, Petrovic I, Braverman LE, et al. Free T4 immunoassays are flawed during pregnancy. Am J Obstet Gynecol. 2009 Mar;200(3):260. e1-6.

17 Anckaert E, Poppe K, Van Uytfanghe K, Schiettecatte J, Foulon W, Thienpont LM. FT4 immunoassays may display a pattern during pregnancy similar to the equilibrium dialysis ID-LC/tandem MS candidate reference measurement procedure in spite of susceptibility towards binding protein alterations. Clin Chim Acta. 2010 Sep;411(17-18):1348-53.

18 Bliddal S, Feldt-Rasmussen U, Boas M, Faber J, Juul A, Larsen T, et al. Gestational age-specific reference ranges from different laboratories misclassify pregnant women's thyroid status: comparison of two longitudinal prospective cohort studies. Eur J Endocrinol. 2013 Dec;170(2):329-39.

19 Korevaar TI, Chaker L, Medici M, de Rijke YB, Jaddoe VW, Steegers EA, et al. Maternal total T4 during the first half of pregnancy: physiologic aspects and the risk of adverse outcomes in comparison with free T4. Clin Endocrinol (Oxf). 2016 Nov;85(5):757-63.

20 Andersen SL, Olsen J, Laurberg P. Foetal programming by maternal thyroid disease. Clin Endocrinol (Oxf). 2015 Dec;83(6):751-8.

21 Andersen SL, Carlé A, Karmisholt J, Pedersen IB, Andersen S. MECHANISMS IN ENDOCRINOLOGY: Neurodevelopmental disorders in children born to mothers with thyroid dysfunction: evidence of fetal programming? Eur J Endocrinol. 2017 Jul;177(1):R27-36.

22 Laurberg P, Andersen SL, Pedersen IB, Andersen S, Carlé A. Screening for overt thyroid disease in early pregnancy may be preferable to searching for small aberrations in thyroid function tests. Clin Endocrinol (Oxf). 2013 Sep;79(3):297-304.
23 Haddow JE, Palomaki GE, Allan WC, Williams JR, Knight GJ, Gagnon J, et al. Maternal thyroid deficiency during pregnancy and subsequent neuropsychological development of the child. N Engl J Med. 1999 Aug;341(8): 549-55.

24 Andersen SL, Andersen S, Liew Z, Vestergaard $\mathrm{P}$, Olsen J. Maternal thyroid function in early pregnancy and neuropsychological performance of the child at 5 years of age. J Clin Endocrinol Metab. 2018 Feb;103(2):660-70.

25 Bava S, Ballantyne AO, Trauner DA. Disparity of verbal and performance IQ following early bilateral brain damage. Cogn Behav Neurol. 2005 Sep;18(3):163-70.

26 Gläscher J, Tranel D, Paul LK, Rudrauf D, Rorden C, Hornaday A, et al. Lesion mapping of cognitive abilities linked to intelligence. Neuron. 2009 Mar;61(5):681-91.

27 Jansen TA, Korevaar TIM, Mulder TA, White T, Muetzel RL, Peeters RP, et al. Maternal thyroid function during pregnancy and child brain morphology: a time window-specific analysis of a prospective cohort. Lancet Diabetes Endocrinol. 2019 Aug;7(8):629-37.

28 Cooper DS, Biondi B. Subclinical thyroid disease. Lancet. 2012 Mar;379(9821):1142-54.

29 Fraenkel M, Shafat T, Cahn A, Erez O, Novack $\mathrm{V}$, Tsur $\mathrm{A}$. Low thyroid-stimulating hormone and its persistence beyond the first trimester of pregnancy. Int J Gynaecol Obstet. 2018 Sep;142(3):270-6.

30 Fan J, Zhang Y, Zhang C, Barjaktarovic M, Yang X, Peeters RP, et al. Persistency of Thyroid Dysfunction from Early to Late Pregnancy. Thyroid. 2019 Oct;29(10):1475-84.

31 Andersen SL, Olsen J. Early Pregnancy Thyroid Function Test Abnormalities in Biobank Sera from Women Clinically Diagnosed with Thyroid Dysfunction Before or After Pregnancy. Thyroid. 2017 Mar;27(3):451-9.

32 Knøsgaard L, Andersen S, Hansen AB, Vestergaard P, Andersen SL. Thyroid function abnormalities and thyroid autoantibodies in Danish pregnant women. Clin Endocrinol (Oxf). 2019. DOI: 10.1111/cen.14147.

33 Selmer C, Faber J. Editorial commentary: subclinical thyroid dysfunction and cardiovascular risk: nothing to lose, everything to gain? Trends Cardiovasc Med. 2019. DOI: 10.1016/j. tcm.2019.05.002.

34 Casey BM, Dashe JS, Wells CE, McIntire DD, Leveno KJ, Cunningham FG. Subclinical hyperthyroidism and pregnancy outcomes. Obstet Gynecol. 2006 Feb;107(2 Pt 1):337-41. 
35 Korevaar TI, Derakhshan A, Taylor PN, Meima M, Chen L, Bliddal S, et al.; Consortium on Thyroid and Pregnancy Study Group on Preterm Birth. Association of Thyroid Function Test Abnormalities and Thyroid Autoimmunity with Preterm Birth: A Systematic Review and Meta-analysis. JAMA. 2019 Aug; 322(7):632-41.

36 Negro R, Formoso G, Mangieri T, Pezzarossa A, Dazzi D, Hassan H. Levothyroxine treatment in euthyroid pregnant women with autoimmune thyroid disease: effects on obstetrical complications. J Clin Endocrinol Metab. 2006 Jul;91(7):2587-91.

37 Negro R, Schwartz A, Gismondi R, Tinelli A, Mangieri T, Stagnaro-Green A. Universal screening versus case finding for detection and treatment of thyroid hormonal dysfunction during pregnancy. J Clin Endocrinol Metab. 2010 Apr;95(4):1699-707.

38 Nazarpour S, Ramezani Tehrani F, Simbar M, Tohidi M, Alavi Majd H, Azizi F. Effects of levothyroxine treatment on pregnancy outcomes in pregnant women with autoimmune thyroid disease. Eur J Endocrinol. 2017 Feb; 176(2):253-65.

39 Lazarus JH, Bestwick JP, Channon S, Paradice R, Maina A, Rees R, et al. Antenatal thyroid screening and childhood cognitive function. N Engl J Med. 2012 Feb;366(6):493-501.

40 Casey BM, Thom EA, Peaceman AM, Varner MW, Sorokin Y, Hirtz DG, et al.; Eunice Kennedy Shriver National Institute of Child Health and Human Development MaternalFetal Medicine Units Network. Treatment of Subclinical Hypothyroidism or Hypothyroxinemia in Pregnancy. N Engl J Med. 2017 Mar;376(9):815-25.

41 Wang $\mathrm{H}$, Gao H, Chi H, Zeng L, Xiao W, Wang Y, et al. Effect of Levothyroxine on Miscarriage among Women with Normal Thyroid Function and Thyroid Autoimmunity Undergoing in vitro Fertilization and Embryo Transfer: A Randomized Clinical Trial. JAMA. 2017 Dec;318(22):2190-8.

42 Nazarpour S, Ramezani Tehrani F, Simbar M, Tohidi M, Minooee S, Rahmati M, et al. Effects of Levothyroxine on Pregnant Women with Subclinical Hypothyroidism, Negative for Thyroid Peroxidase Antibodies. J Clin Endocrinol Metab. 2018 Mar;103(3):926-35.

43 Dhillon-Smith RK, Middleton LJ, Sunner KK, Cheed V, Baker K, Farrell-Carver S, et al. Levothyroxine in Women with Thyroid Peroxidase Antibodies before Conception. N Engl J Med. 2019 Apr;380(14):1316-25.

44 Brent GA. The debate over thyroid-function screening in pregnancy. N Engl J Med. 2012 Feb;366(6):562-3.

45 Cooper DS, Pearce EN. Subclinical Hypothyroidism and Hypothyroxinemia in Pregnancy - Still No Answers. N Engl J Med. 2017 Mar; 376(9):876-7.
46 Hales C, Taylor PN, Channon S, Paradice R, McEwan K, Zhang L, et al. Controlled Antenatal Thyroid Screening II: effect of treating maternal sub-optimal thyroid function on child cognition. J Clin Endocrinol Metab. 2018 Apr;103(4):1583-91.

47 Hales C, Taylor PN, Channon S, McEwan K, Thapar A, Langley K, et al. Controlled Antenatal Thyroid Screening II: effect of treating maternal sub-optimal thyroid function on child behaviour. J Clin Endocrinol Metab. 2019 Oct;dgz098.

48 Maraka S, Mwangi R, McCoy RG, Yao X, Sangaralingham LR, Singh Ospina NM, et al. Thyroid hormone treatment among pregnant women with subclinical hypothyroidism: US national assessment. BMJ. 2017 Jan;356:16865.

49 Man EB. Thyroid function in pregnancy and infancy. Maternal hypothyroxinemia and retardation of progeny. CRC Crit Rev Clin Lab Sci. 1972 Jun;3(2):203-25.

50 Sinha AK, Pickard MR, Ekins RP. Maternal hypothyroxinemia and brain development: I. A hypothetical control system governing fetal exposure to maternal thyroid hormones. Acta Med Austriaca. 1992;19 Suppl 1:40-8.

51 Glinoer D, Delange F. The potential repercussions of maternal, fetal, and neonatal hypothyroxinemia on the progeny. Thyroid. 2000 Oct;10(10):871-87.

52 Morreale de Escobar G, Obregón MJ, Escobar del Rey F. Is neuropsychological development related to maternal hypothyroidism or to maternal hypothyroxinemia? J Clin Endocrinol Metab. 2000 Nov;85(11):3975-87.

53 Morreale de Escobar G. Maternal hypothyroxinemia versus hypothyroidism and potential neurodevelopmental. Alterations of her offspring. Ann Endocrinol (Paris). 2003 Feb; 64(1):51-2.

54 Román GC. Autism: transient in utero hypothyroxinemia related to maternal flavonoid ingestion during pregnancy and to other environmental antithyroid agents. J Neurol Sci. 2007 Nov;262(1-2):15-26.

55 Negro R, Soldin OP, Obregon MJ, StagnaroGreen A. Hypothyroxinemia and pregnancy. Endocr Pract. 2011 May-Jun;17(3):422-9.

56 Henrichs J, Ghassabian A, Peeters RP, Tiemeier $\mathrm{H}$. Maternal hypothyroxinemia and effects on cognitive functioning in childhood: how and why? Clin Endocrinol (Oxf). 2013 Aug;79(2):152-62.

57 Furnica RM, Lazarus JH, Gruson D, Daumerie C. Update on a new controversy in endocrinology: isolated maternal hypothyroxinemia. J Endocrinol Invest. 2015 Feb;38(2): 117-23.

58 Chan S, Boelaert K. Optimal management of hypothyroidism, hypothyroxinaemia and euthyroid TPO antibody positivity preconception and in pregnancy. Clin Endocrinol (Oxf). 2015 Mar;82(3):313-26.

59 Lazarus JH, Taylor PN. Hypothyroxinaemia and brain development. Acta Endocrinol (Bucur). 2016 Jan-Mar;12(1):1-6.
60 Min H, Dong J, Wang Y, Wang Y, Teng W, Xi Q, et al. Maternal Hypothyroxinemia-Induced Neurodevelopmental Impairments in the Progeny. Mol Neurobiol. 2016 Apr;53(3): 1613-24.

61 Dosiou C, Medici M. MANAGEMENT OF ENDOCRINE DISEASE: Isolated maternal hypothyroxinemia during pregnancy: knowns and unknowns. Eur J Endocrinol. 2017 Jan; 176(1):R21-38.

62 Boyages SC, Halpern JP. Endemic cretinism: toward a unifying hypothesis. Thyroid. 1993; 3(1):59-69.

63 Pharoah PO, Connolly KJ, Ekins RP, Harding AG. Maternal thyroid hormone levels in pregnancy and the subsequent cognitive and motor performance of the children. Clin Endocrinol (Oxf). 1984 Sep;21(3):265-70.

64 Pop VJ, Kuijpens JL, van Baar AL, Verkerk G, van Son MM, de Vijlder JJ, et al. Low maternal free thyroxine concentrations during early pregnancy are associated with impaired psychomotor development in infancy. Clin Endocrinol (Oxf). 1999 Feb;50(2):149-55.

65 Aung T, Halsey J, Kromhout D, Gerstein HC, Marchioli R, Tavazzi L, et al. Associations of Omega-3 Fatty Acid Supplement Use with Cardiovascular Disease Risks: Meta-analysis of 10 Trials Involving 77917 Individuals. JAMA Cardiol. 2018 Mar;3(3):225-34.

66 Manson JE, Cook NR, Lee IM, Christen W, Bassuk SS, Mora S, et al.; VITAL Research Group. Vitamin D Supplements and Prevention of Cancer and Cardiovascular Disease. N Engl J Med. 2019 Jan;380(1):33-44.

67 Vandenbroucke JP, Merrall EL. The HRT controversy: observational studies and RCTs fall in line. Lancet. 2009 Apr;373(9671):12335.

68 Jepsen P, Johnsen SP, Gillman MW, Sørensen HT. Interpretation of observational studies. Heart. 2004 Aug;90(8):956-60.

69 Knight BA, Shields BM, Hattersley AT, Vaidya B. Maternal hypothyroxinaemia in pregnancy is associated with obesity and adverse maternal metabolic parameters. Eur J Endocrinol. 2016 Jan;174(1):51-7.

70 Haddow JE, Craig WY, Neveux LM, Palomaki GE, Lambert-Messerlian G, Malone FD, et al.; First and Second Trimester Risk of Aneuploidy (FaSTER) Research Consortium. Free Thyroxine During Early Pregnancy and Risk for Gestational Diabetes. PLoS One. 2016 Feb; 11(2):e0149065.

71 Teng X, Shan Z, Li C, Yu X, Mao J, Wang W, et al. Iron Deficiency May Predict Greater Risk for Hypothyroxinemia: A Retrospective Cohort Study of Pregnant Women in China. Thyroid. 2018 Aug;28(8):968-75.

72 Ellervik C, Roselli C, Christophersen IE, Alonso A, Pietzner M, Sitlani CM, et al. Assessment of the Relationship Between Genetic Determinants of Thyroid Function and Atrial Fibrillation: A Mendelian Randomization Study. JAMA Cardiol. 2019 Feb;4(2): $144-52$. 